\section{Cas clinique Klinischer Fall}

\section{Présentation inhabituelle de la syphilis secondaire}

\section{F. Scolari, Genève}

Depuis peu, la syphilis fait une réapparition dans nos consultations de vénéréologie, probablement à cause d'une forte augmentation des cas dans les pays de l'est européen dans lesquels le tourisme sexuel est important. Comme toujours, cette maladie peut se présenter sous des formes cliniques inhabituelles et trompeuses, qui lui ont valu la réputation de «grande simulatrice». Ce cas clinique en est un exemple.

\section{Observation}

C.A. est un homme de 54 ans, suivi par un rhumatologue pour de multiples douleurs articulaires chroniques et par un neurologue pour des paresthésies de la paume de la main gauche. Ces douleurs, probablement en partie liées à un état dépressif, ont motivé la prise de nombreux anti-inflammatoires non stéroïdiens. Un mois après l'introduction du célécoxibe, dernier de la liste, sont apparues les lésions cutanées qui ont motivé la consultation dermatologique. Elles étaient localisées uniquement sur le scrotum, asymptomatiques, et leur appari-

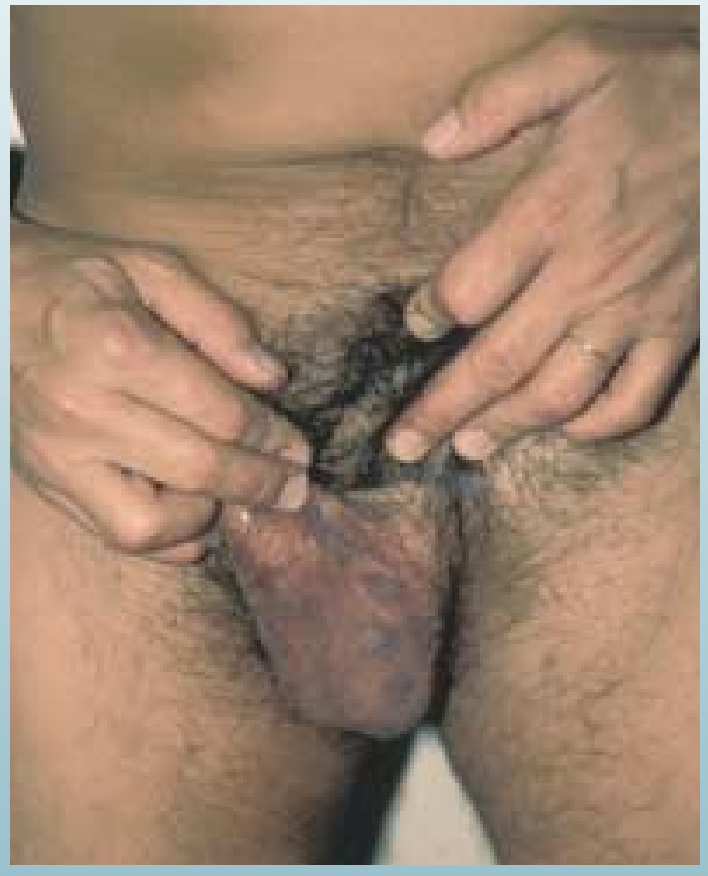

Fig. la

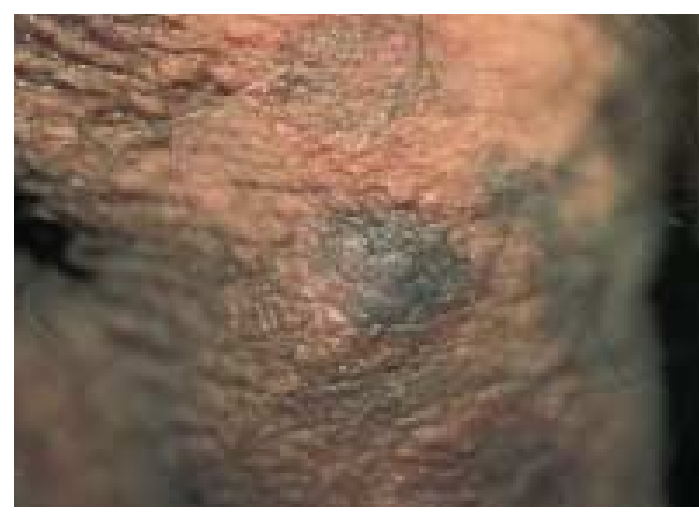

Fig. 1b

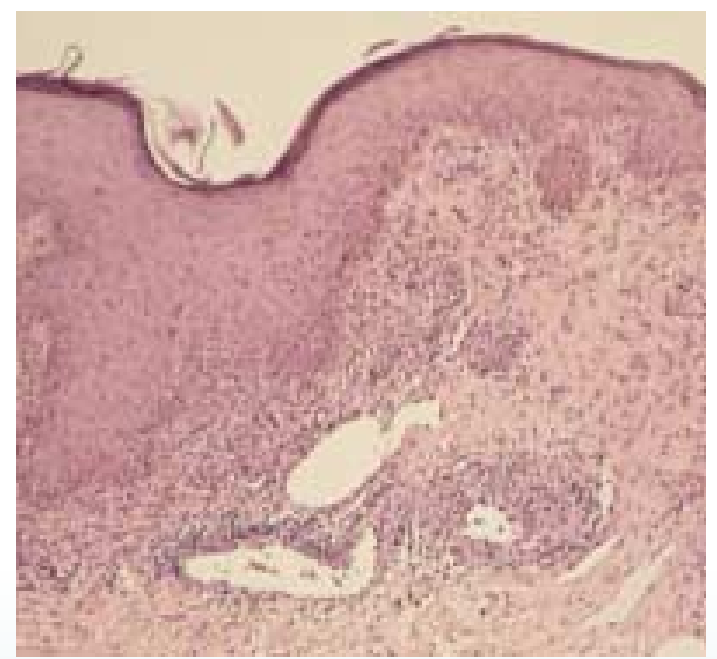

Fig. 2

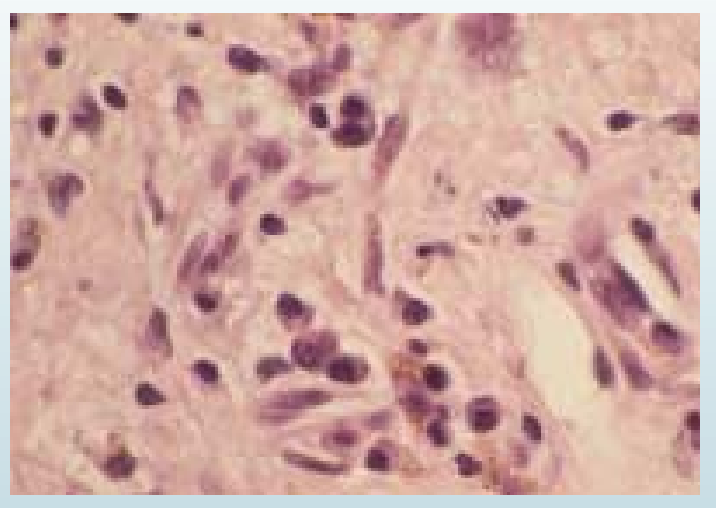

Fig. 3

tion n'avait pris que quelques jours, aux dires du patient. Cliniquement on observait des macules infiltrées, bleuâtres, assez bien délimitées, de la taille de quelques centimètres avec, par endroits, une petite atteinte épidermique (fig. 1a, b). Tenant compte de l'anamnèse et du contexte clinique, on évoque la possibilité d'une toxidermie médicamenteuse à type d'érythème pigmenté fixe: une biopsie est toutefois pratiquée, afin d'étayer le diagnostic. L'aspect histologique n'est pas incompatible avec le diagnostic proposé, car il montre des éléments qui suggèrent une dermo-épidermite lichénoïde (fig. 2). Néanmoins, l'histopathologiste attire l'attention sur la présence de plasmocytes (fig. 3) qui posent le diagnostic différentiel d'une 


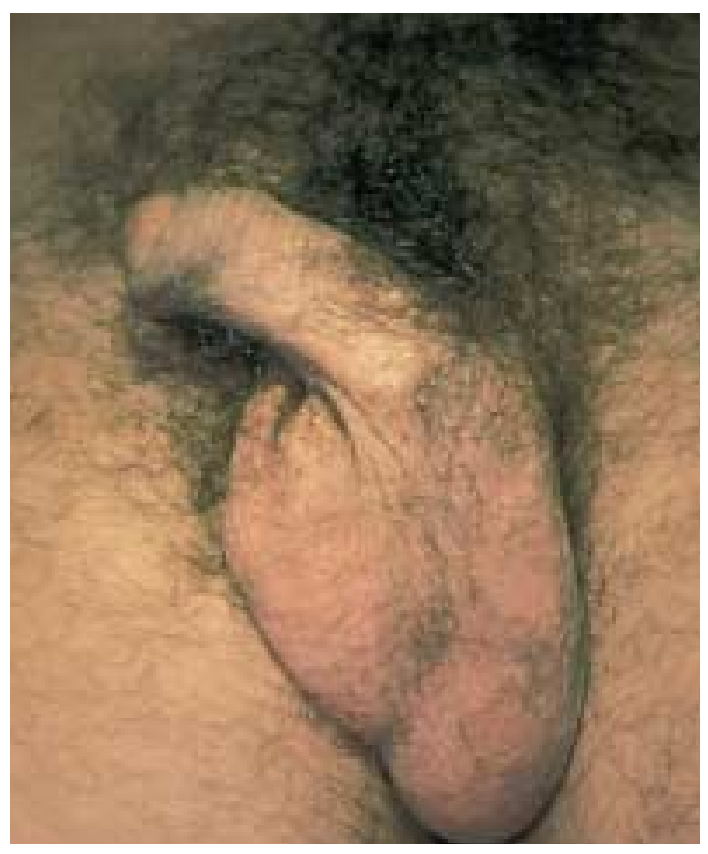

syphilis secondaire. Une sérologie luétique est alors demandée et elle se révèle fortement positive (VDRL 1:64, TPHA 1:20 480, FTAabs positif), confirmant ainsi le diagnostic. La sérologie pour le VIH est négative. Le patient avoue une probable contamination par contact homosexuel. Une hospitalisation dans le service de neurologie, déjà prévue par le neurologue, nous permet de pratiquer une ponction lombaire et d'exclure une atteinte du système nerveux central par le tréponème pâle. L'administration de trois injections hebdomadaires de 2,4 MU de benzathine-pénicilline permet une guérison complète (fig. 4).

\section{Discussion}

Il s'agit ici d'une présentation très particulière de la syphilis, absolument pas caractéristique. Des formes nodulaires et infiltrées, pouvant même simuler un lymphome, ont été rapportées chez des sujets infectés aussi par le VIH. Mais les lésions, même si peu nombreuses, étaient toujours disséminées sur tout le corps. Ce cas illustre bien aussi l'importance des examens complémentaires et, notamment, de l'examen histologique, qu'il ne faut pas hésiter à demander dans les cas où la clinique est douteuse. 